Rheumatology clinic, with variable waiting times causing potential delay in starting treatment such as DMARDs. There is also a cost implication with respect to commissioning of the Radiology scan appointments. In Bolton, an US system was installed in Rheumatology from 2016 to perform inclinic US, for improving access and reducing referrals to Radiology.

Objectives: We aimed to assess the US usage pattern before and after the new Rheumatology US was installed, with respect to access based on age and gender, the pattern of scanning in-clinic, and rate of synovitis diagnsosis, using a quality improvement framework.

Methods: US referral data from the Radiology department from October 2015 to March 2016 were collated as baseline on a MS Access database. Two Consultant Rheumatologists performed US in-clinic. From May to October 2016 Rheumatology in-clinic data, and also Radiology referral data were collated on the database for comparison. Patient demographics and diagnoses were collated from clinic letters. Descriptive statistics were processed in MS Excel 2010.

Results: Between October 2015 and March 2016, 68 patients (median age 52 yrs; 28\% male) had scans in Radiology. Between May and October 2016, 59 patients (median age $60 \mathrm{yrs;} 21 \%$ male) had scans in Radiology, and 78 patients (median age 59yrs; 35\% male) had scans in Rheumatology clinic. There was no significant difference in scanned areas amongst the three cohorts with the most common overall being hand/wrist area $(n=57,54$ and 61 respectively; total $84 \%)$, followed by foot/ankle ( $n=6,4$ and 4 respectively; total $7 \%$ ). Between the two time periods, there was an increased trend in final working diagnoses of inflammatory arthritis (28 [41\%] vs 36 [61\%] and 39 [50\%] in the 3 cohorts respectively); and a decreasing trend in the final working diagnoses of noninflammatory conditions (OA, FM, other non-inflammatory pain: 36 [53\%] vs 22 [37.3\%] and 28 [35.9\%] in the 3 cohorts respectively).

Conclusion: The availability of Rheumatology US was associated with increased propensity for scanning older patients and a greater proportion of men compared to previously, a qualitative improvement as synovitis diagnosis could be delayed in these groups. The introduction of Rheumatology US was also associated with a trend towards higher proportion of inflammatory diagnoses, suggesting potentially increased appropriate clinical use due to availabiltiy in-clinic. This is supported by the majority being hand/wrist scans, suggesting Rheumatology use is mainly aimed at diagnosing synovitis. Although there was a modest reduction of $13 \%$ in Radiology referrals between the two time periods, there was an overall increase of scans performed by $101 \%$ following introduction of the Rheumatology US service. We therefore recommend Rheumatology in-clinic US provision, as in addtion to obvious improvement in time to diagnosis, it is likely to increase the range of patients able to access US eg older patients and men, and also likely to increase the pick-up rate of synovitis by improving patient selection.

Disclosure of Interests: None declared

DOI: 10.1136/annrheumdis-2019-eular.7465

\section{AB1188 AUDIT: IMPACT OF MUSCULOSKELETAL ULTRASOUND USE IN RHEUMATOLOGY CLINICS}

Andrew Wilkinson, Mike Reed, Sara Else. Forth Valley Royal Hospital, Larbert, United Kingdom

Background: Musculoskeletal ultrasound (MSUS) has assumed a prominent role in rheumatological practice as both a diagnostic and monitoring tool. ${ }^{1}$ It has utility in excluding and quantifying active synovitis and can be used to guide adjustments of therapies. . $^{2,3}$

Objectives: We aimed to observe impacts on clinical practice of utilising ultrasound (US) scanners in outpatient clinics in a district general rheumatology service. We were particularly interested to see if this affected treatment choices, follow-up plans and referrals for radiological investigations.

Methods: US scanners were obtained and used by 2 rheumatology consultants with previous ultrasound training. Scanning was performed during standard 20 minute appointments within general rheumatology outpatient clinics. No extra time was allocated. Over 8 weeks, we completed questionnaires for each scan and assessed the impact on management decisions.

Results: Over 8 weeks, data was collected for 36 consecutive patients scanned. Commonly imaged joints were hands $(81 \%, n=29)$, wrists $(75 \%$, $n=27)$, feet $(19 \%, n=7)$, and ankles $(14 \%, n=5)$.

Most common patient diagnoses were osteoarthritis $(n=13,36 \%)$, rheumatoid arthritis $(n=10,28 \%)$ and psoriatic arthropathy $(n=6,17 \%)$. The remaining patients had diagnostic labels including undifferentiated inflammatory arthritis or no formal diagnosis. Abnormalities suggesting active inflammation were seen in $42 \%$. In $33 \%$ osteoarthritic changes were observed, erosions were seen in $5 \% .20 \%$ scans were normal.

US altered the management in $58 \%(n=21 / 36)$ of cases. These alterations to management included: drug added $(n=5)$, drug stopped $(n=3)$, dose increase $(n=1)$, joint injection $(n=3)$, intramuscular injection $(n=2)$. Of the medications added, 3 were DMARDs, 1 was a biologic therapy and one case was not specified. The medications stopped were all DMARDS.

US use in clinic prevented radiological investigation in $72 \%(26 / 36)$ of cases. These were radiology ultrasound $(n=23)$ and MRI $(n=3)$. Hands and wrists scans accounted for $86 \%$ of these.

US altered the follow up in $56 \%(20 / 36)$ of cases. Of these, $40 \%(8 / 20)$ were reviewed earlier, and $40 \%(8 / 20)$ of patients were discharged based on ultrasound information.

In 16/36 patients, US did not alter management. The average time taken to scan in clinic was 7 minutes per patient. The median time was 5 minutes, with the average skewed due to a few prolonged scans of multiple anatomical areas.

Conclusion: This audit has demonstrated that US use has impacted on our clinical practice. We identified patients to be seen earlier and altered management based on US findings.

US use also prevented radiological investigations in $72 \%$ of cases. This has positive impacts on staffing, finances, radiology department capacity and patient convenience. Furthermore, we were able to discharge $40 \%$ of patients who otherwise would have been recalled.

Overall, we believe this audit shows positive impacts of MSUS on patient care. Challenges include time pressures of scanning in clinic, time and cost implications of ultrasound training and the need to standardise and record ultrasound methods and findings consistently. Costs are offset by savings to radiology resources and increased discharges. We plan to expand ultrasound usage and train our colleagues to employ ultrasound for our patients.

\section{REFERENCES}

[1] Möller I, Janta I, Backhaus M, et al. The 2017 EULAR standardised proce dures for ultrasound imaging in rheumatology Annals of the Rheumatic Diseases 2017;76:1974-1979.

[2] Alivernini S, et al. Tapering and discontinuation of TNF-alpha blockers without disease relapse using ultrasonography as a tool to identify patients with rheumatoid arthritis in clinical and histological remission. Arthritis Res Ther18:39.

[3] Bawa S, Todd M, France J SAT0203 Optimization of biologic tapering using ultrasound in ra patients Annals of the Rheumatic Diseases 2018;77:962

Disclosure of Interests: Andrew Wilkinson: None declared, Mike Reed Speakers bureau: Paid to speak at meeting by Novartis, Sara Else Consultant for: Contributed to advisory boards for: Abbvie, Norartis, Roche and Chungai Pharma., Speakers bureau: Paid to organise and chair meetings, and speak at educational events by following companies: BMS, Celegene, Grifols, Jansess, MSD, Novartis, UCB.

DOI: 10.1136/annrheumdis-2019-eular.5310

\section{AB1189 THE IMPORTANCE OF POSITRON EMISSION TOMOGRAPHY-COMPUTED TOMOGRAPHY (PET -CT) IN FEVER OF UNKNOWN ORIGIN (FUO) AND INFLAMMATION OF UNKNOWN ORIGIN (IUO)}

Tahir Saygın Öğüt, Veli yazısız, Funda Erbasan, Mustafa Ender Terzioğlu. Akdeniz University, Department of Internal Medicine, Antalya, Turkey

Background: Fever of unknown origin (FUO) and inflammation of unknown origin (IUO) are rare clinical problems but diagnostically challenging for clinicians. Patients are exposed to extensive and expensive medical examinations for the identification of the FUO/IUO. FDG PET-CT can help to identify the underlying disease in these patients.

Objectives: The aim of this study was to evaluate the role of PET-CT in patients with FUO/IUO and to identfy FDG uptake sites and patterns

Methods: Total of 286 patients who were performed 18F FDG-PET-CT between Jan 2014 and Dec 2017 were evaluated, retrospectively. There were a total 62 patients scanned by PET-CT for FUO/IUO. Definitive diagnosis, PET-CT patterns, organ involvement and SUVmax values in these patients were reevaluated

Results: Rheumatic disease in 27 patients (43.5\%), 20 malignancy $(32.3 \%)$ and 6 infectious diseases $(9.7 \%)$ were diagnosed in the FUO IUO patients performed PET-CT scan. Systemic vasculitis were the most frequently diagnosed rheumatic disease. 11 patients of the 62 patients $(17.7 \%)$ were diagnosed large vessel vasculitis (9 Takayasu arteritis, 2 